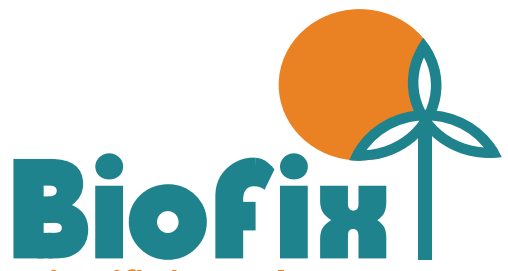

scientific journal

v. 2 n. 12017

Recebido em 24/03/2017

Aceito em 03/04/2017

Publicado em 20/04/2017

DOI: dx.doi.org/10.5380/biofix.v2i1.51395

\section{MODELAGEM DA RELAÇÃO HIPSOMÉTRICA PARA UM POVOAMENTO HÍBRIDO DE EUCALIPTO NA AMAZÔNIA BRASILEIRA}

\author{
MODELING HEIGHT-DIAMETER RELATIONSHIP FOR A HYBRID \\ EUCALYPTUS STAND IN THE BRAZILIAN AMAZON
}

\begin{abstract}
Universidade Federal do Pará, Altamira, Pará, Brasil alex_souza3@hotmail.com ${ }^{1}$ \&deivisonvs@ufpa.com ${ }^{3}$

Universidade Federal do Paraná, Curitiba, Paraná, Brasil joilanxipaia@hotmail.com²
\end{abstract}

\section{RESUMO}

Este trabalho objetivou ajustar modelos hipsométricos tradicionais, para um povoamento híbrido (Eucalyptus urophylla e Eucalyptus grandis) aos oito anos de idade, no município de Pacajá, Pará. Foram estabelecidas quatro unidades de amostras quadradas de $30 \mathrm{~m} \times 30 \mathrm{~m}$, totalizando $3.600 \mathrm{~m}^{2}$ de área amostrada, sob o processo de amostragem inteiramente aleatório. Foram testados 17 modelos hipsométricos matemáticos de natureza logarítmica e aritmética. Os critérios de seleção dos modelos foram: i) significância da estatística $F$ da Análise de Variância (ANOVA) da regressão; ii) coeficiente de determinação ajustado ( $R^{2}$ aj.); iii) erro padrão da estimativa em porcentagem (Syx\%); iv) desvio médio percentual (DMP\%) e v) análise gráfica dos resíduos. A maioria dos modelos apresentou baixos valores de $\mathrm{R}^{2} \mathrm{aj}$., Syx\% e DMP\%, com destaque para o modelo Linear e Assmann. Os modelos Hiperbólico: $V D A P^{2} /(h-1,3)=0,3071+0,2048 D A P$, e Naslund: $D A P / V h=0,3426+0,2103 D A P$ foram mais apropriados para estimarem a altura da árvore em pé para o híbrido, tendo em vista as melhores medidas de precisão.

PALAVRAS-CHAVE: Clones de Eucalyptus, Equações hipsométricas, Modelos tradicionais.
KEYWORDS: Eucalyptus clones, Height-diameter equations, Traditional models.

\section{ABSTRACT}

This study aimed to adjust traditional height-diameter models for an 8-year old hybrid Eucalyptus stand (Eucalyptus urophylla and Eucalyptus grandis) in the municipality of Pacajá, Pará. Four units of $30 \mathrm{~m} \times 30 \mathrm{~m}$ square samples, totaling $3,600 \mathrm{~m}^{2}$ of sampled area, were established under a completely random sampling process. Seventeen logarithmic and arithmetic height-diameter models were tested. The criteria used to select the models were: i) FStatistics significance from variance analysis (ANOVA), and regression; ii) adjusted coefficient of determination ( $R^{2}$ aj.); iii) recalculated standard error of the estimate in percentage (Syx\%); iv) average percentage deviation (APD\%); and v) graphical analysis of the residues. Most of the models presented low values of $R^{2} a j$. Syx\%, and APD\%, with emphasis on the Assmann straight line model. Hyperbolic model: $V D A P^{2} /(h-1.3)=0.3071+0.2048 D A P$, and Naslund model: $D A P / V h=0.3426+0.2103 D A P$ were the most accurate in estimating the height of the standing hybrid trees; however, the hyperbolic model was slightly more suitable because of its lower standard error of the estimate. 


\section{INTRODUÇÃO}

O eucalipto é a principal espécie plantada no Brasil, contando com aproximadamente 5.102.030 hectares de áreas plantadas, abastecendo principalmente as indústrias de madeira, celulose, papel, compensado, e siderúrgicas do país. O estado do Pará até o ano de 2012 estava em 9o lugar entre os estados brasileiros com plantios de eucaliptos, compreendendo uma área de 159.657 ha, representando $3,13 \%$ do território nacional (ABRAF, 2013).

Nas últimas décadas, diversos pesquisadores têm despendido esforços em estudar o gênero Eucalyptus sob diversos aspectos e diversificadas condições edafoclimáticas, dada a sua larga escala de produção e diversidade de utilização. Dentre os inúmeros estudos desenvolvidos destacam-se àqueles destinados a modelagem da relação hipsométrica (SOARES et al., 2004; RIBEIRO et al., 2010; RUFINO et al., 2010; OLIVEIRA et al., 2011; SOUSA et al., 2013), através do ajuste de modelos matemáticos tradicionais ou genéricos.

Os modelos hipsométricos são equações ajustadas que expressam a relação altura-diâmetro da árvore (SANQUETTA et al., 2009), através dos quais é possível obter estimativas acuradas da altura da árvore. A medição da altura em povoamentos florestais é de grande importância para a estimava do volume e qualificação da produtividade em determinado local, sendo fundamental no planejamento da produção florestal (RIBEIRO et al., 2010).

A modelagem hipsométrica é um aspecto importante a ser considerado nos inventários florestais, tendo em vista ser a mensuração indireta da altura da árvore uma atividade morosa e com elevada margem de erro na coleta da informação (SOUSA et al., 2013) e, portanto, altamente dispendiosa.

De acordo com Batista et al. (2014) os padrões hipsométricos diferem para distintos tipos florestais, bem como numa mesma tipologia florestal para distintos sítios, e ainda, dentro de um sitio, em virtude da heterogeneidade ambiental e estrutural, haverá divergentes relações hipsométricas. Nesse contexto, torna-se imperiosa a seleção de modelos mais adequados para estimar a altura de cada povoamento plantado, tendo em vista que existem equações que melhor se adaptam a cada povoamento (OLIVEIRA et al., 2011), reduzindo sobremaneira os custos do inventário florestal (BARROS et al., 2002).

Este trabalho teve por objetivo ajustar modelos hipsométricos tradicionais para um povoamento do híbrido (E. urophylla e E. grandis) na Amazônia brasileira, com fins de indicar aquele(s) que melhor descreva(m) a relação altura-diâmetro.

\section{MATERIAL E MÉTODOS}

\section{Localização e caracterização da área de estudo}

O estudo foi conduzido em um povoamento híbrido ( $E$. urophylla e $E$. grandis) com idade de oito anos, estabelecido sob o espaçamento $3 \mathrm{~m}$ x $3 \mathrm{~m}$. O povoamento pertence à empresa Ipiaçava Indústria e Comércio de Madeiras LTDA, com área total de 13,3629 hectares, localizado às margens da BR-230 (Rodovia Transamazônica), na mesorregião do Sudoeste paraense, Pacajá, Pará, sob coordenadas Latitude: N -03 $38^{\prime}$ 59,20473" e Longitude: E -50 57' 19,45084"(Datum WGS 84).

A região possui clima tropical úmido do tipo $A f$, conforme a classificação de Köeppen, com estações quentes e chuvosas, caracterizado por umidade relativa do ar de $85 \%$, temperatura anual variando de $21^{\circ} \mathrm{C}$ a $32^{\circ} \mathrm{C}$ e índice pluviométrico anual de $2.300 \mathrm{~mm}$, sendo mais frequente nos meses de fevereiro, março e abril. Em agosto, setembro e outubro as chuvas diminuem, formando o período mais seco (IDESP, 2011). O solo é caracterizado como Argissolo Vermelho-Amarelo Distrófico de textura argilosa/muito argilosa e médioargilosa (IBGE, 2012).

\section{Sistema de amostragem}

Os dados utilizados neste estudo foram obtidos de um povoamento plantado do híbrido (E. urophylla e $E$. grandis). Utilizou-se o processo de amostragem inteiramente aleatório, com uso de 4 (quatro) unidades de amostras quadradas de $30 \mathrm{~m} \times 30 \mathrm{~m}$, totalizando 3.600 $\mathrm{m}^{2}$ de área amostrada. Foram medidas 328 árvoresamostras, cuja distribuição por classes de diâmetro e altura, é apresentada na Tabela 1.

Do total de 328 árvores-amostras, 50 árvores distribuídas nas diferentes classes diamétricas e altura foram separadas em um banco de dados independente, compondo uma amostra para validação do modelo de regressão selecionado. Foram mensuradas in loco as seguintes variáveis: i) diâmetro a $1,3 \mathrm{~m}$ do solo (DAP), medido com fita diamétrica; e ii) altura total $(h)$, medida com o auxílio do hipsômetro de Häglof, ajustado para a escala de distância de $10 \mathrm{~m}$. 
Tabela 1. Número de indivíduos, por classes de diâmetro e altura, das 328 árvores-amostras

\begin{tabular}{ccccccc}
\hline Classe de & \multicolumn{5}{c}{ Classe de altura $(\mathbf{m})$} & \multirow{2}{*}{ Total } \\
\cline { 2 - 5 } DAP $(\mathbf{c m})$ & $\mathbf{1 2}+\mathbf{1 5}$ & $\mathbf{1 5}+\mathbf{1 8}$ & $\mathbf{1 8}+\mathbf{2 1}$ & $\mathbf{2 1}+\mathbf{2 4}$ & \\
\hline $4 \vdash 7$ & 4 & 2 & - & - & 6 \\
\hline $7 \vdash 10$ & 1 & 26 & 10 & - & 37 \\
$10+13$ & - & 13 & 56 & - & 69 \\
$13 \vdash 16$ & - & - & 88 & 6 & 94 \\
$16+19$ & - & - & 73 & 18 & 91 \\
\hline $19+22$ & - & - & 22 & 6 & 28 \\
\hline $22+25$ & - & - & 2 & 1 & 3 \\
\hline Total & 5 & 41 & 251 & 31 & $\mathbf{3 2 8}$ \\
\hline
\end{tabular}

\section{Modelos hipsométricos}

Foram avaliados 17 modelos tradicionais (aritméticos e logarítmicos) obtidos na literatura (Tabela 2), com intuito de selecionar aquele(s) mais acurado(s) para descrever a relação altura-diâmetro.

Tabela 2. Modelos hipsométricos tradicionais testados

\begin{tabular}{|c|c|c|}
\hline & Modelos Aritméticos & Autor \\
\hline 1 & $h=\beta_{0}+\beta_{1} D A P+\varepsilon_{i}$ & Linear \\
\hline 2 & $\sqrt{ }(1 / h)=\beta_{0}+\beta_{1} 1 / D A P+\varepsilon_{i}$ & Petterson \\
\hline 3 & 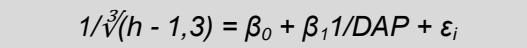 & Petterson \\
\hline 4 & $h=\beta_{0}+\beta_{1} \operatorname{Ln}(D A P)+\varepsilon_{i}$ & Henriksen (1950) \\
\hline 5 & $h=\beta_{0}+\beta_{1} 1 / D A P^{2}+\varepsilon_{i}$ & Assmann \\
\hline 6 & $h=\beta_{0}+\beta_{1} 1 / D A P+\varepsilon_{i}$ & Curtis (1967) \\
\hline 7 & $\sqrt{ } D A P^{2} /(h-1,3)=\beta_{0}+\beta_{1} D A P+\varepsilon_{i}$ & Naslund \\
\hline 8 & $D A P / \sqrt{ } h=\beta_{0}+\beta_{1} D A P+\varepsilon_{i}$ & Hiperbólico \\
\hline 9 & $h=\beta_{0}+\beta_{1} D A P+\beta_{2} D A P^{2}+\varepsilon_{i}$ & Trorey (1932) \\
\hline 10 & $D A P^{2} /(h-1,3)=\beta_{0}+\beta_{1} D A P+\beta_{2} D A P^{2}+\varepsilon_{i}$ & Prodan \\
\hline \multirow[t]{2}{*}{11} & $D A P^{2} / h=\beta_{0}+\beta_{1} D A P+\beta_{2} D A P^{2}+\varepsilon_{i}$ & Prodan \\
\hline & Modelos Logarítmicos & \\
\hline 12 & $\operatorname{Ln}(h-1,3)=\beta_{0}+\beta_{1} 1 / D A P+\varepsilon_{i}$ & Pollanschutz \\
\hline 13 & $\operatorname{Ln}(h)=\beta_{0}+\beta_{1} \operatorname{Ln}(D A P)+\varepsilon_{i}$ & Stoffels \\
\hline 14 & $\operatorname{Ln}(h)=\beta_{0}+\beta_{1} 1 / D A P+\varepsilon_{i}$ & Curtis \\
\hline 15 & $\sqrt[3]{(1 / L n h)=\beta_{0}+\beta_{1} 1 / D A P+\varepsilon_{i}}$ & Petterson \\
\hline 16 & $\operatorname{Ln}(h)=\beta_{0}+\beta_{1} \log (D A P)+\varepsilon_{i}$ & Stoffels \\
\hline 17 & $\operatorname{Ln}(h)=\beta_{0}+\beta_{1} \operatorname{Ln}(D A P)+\beta_{2}(1 / D A P)+\varepsilon_{i}$ & Silva (1980) \\
\hline
\end{tabular}

$h=$ altura total da árvore $(\mathrm{m}) ; D A P=$ diâmetro medido a $1,3 \mathrm{~m}$ do solo $(\mathrm{cm}) ; L n=$ logaritmo neperiano; $\log =$ Logaritmo; $\beta_{0}, \beta_{1} \mathrm{e}$ $\beta_{2}=$ coeficientes da regressão; e $\varepsilon_{i}=$ erro aleatório.

Fonte: Donadoni et al. (2010), Rocha et al. (2010), Soares et al. (2004), Sousa et al. (2013), Azevedo et al. (2011), Curto et al. (2014), Araújo et al. (2012a) e Araújo et al. (2012b).

\section{Critérios estatísticos para a seleção do melhor modelo}

Preliminarmente ao ajuste dos modelos hipsométricos realizou-se a análise exploratória dos dados (AED) através da estatística descritiva, visando identificar possíveis erros na medição das variáveis diâmetro a $1,30 \mathrm{~m}$ do solo (DAP) e altura total (h) e, ainda, detectar potenciais outliers, advindos do processo de medição, ou mesmo, devido às variações biológicas inerentes a cada espécie florestal e de fatores ambientais intrínsecos ao povoamento. Além disso, foi realizado o exame da matriz de correlação entre as variáveis dependentes e independentes e entre as independentes dos modelos selecionados para o ajuste, com fins de avaliar o grau de associação entre as variáveis do modelo.

Para a seleção da melhor equação hipsométrica analisou-se os seguintes critérios estatísticos: i) significância da estatística $F$ da Análise de Variância (ANOVA) da regressão; ii) coeficiente de determinação ajustado ( $R^{2}$ aj.); iii) erro padrão da estimativa recalculado (Syxrecalc.); iv) erro padrão da estimativa recalculado, em porcentagem (Syx\%); v) desvio médio percentual (DMP\%); vi) análise gráfica dos resíduos. A análise de resíduos foi realizada plotando-se o erro residual em função do diâmetro a 1,30 $\mathrm{m}$ do solo (DAP). Além disso, avaliou-se a significância estatística dos coeficientes da regressão através do teste $t$.

A estatística $F$ obtida por meio da análise de variância da regressão tem por objetivo testar a hipótese global da regressão, isto é, de existência ou não da regressão. De acordo com Valente et al. (2011), quando o valor do teste $\mathrm{F}$ for maior que o $\mathrm{F}$ tabelado, rejeita-se a hipótese de não haver regressão, isto é, o modelo é significativo a determinado nível de significância, e conclui-se que pelo menos uma variável independente esteja relacionada com o valor da variável dependente.

Quando a variável dependente sofre algum tipo de transformação, faz-se necessário o recálculo do Syx, para que se possa comparar as equações transformadas com àquelas não transformadas. O recalculo do erro padrão de estimativa foi feito por $\operatorname{Syx}_{(\text {recalc. })}=\sqrt{\sum\left(y_{i}-\hat{y}_{i}\right) /(n-p)}$ em que: Syx(recalc.) = erro padrão da estimativa recalculado; $y_{i}=$ valor real de cada observação; $\hat{y}_{i}=$ valor estimado de cada observação; $n=$ número de dados; e $p=$ número de coeficientes de cada equação.

Além disso, quando a variável dependente sofre uma transformação logarítmica, foi necessário recalcular os valores de volume estimados da variável dependente para corrigir a discrepância, através do Fator de Correção de 
Meyer (FM), determinado através da seguinte formula:

$\mathrm{FM}=e^{0,5\left(\mathrm{Syx}^{2}\right)}$

Em que: $e=$ constante de Euler (2,718281828...); e Syx = o erro padrão de estimativa.

A estatística Fator de Inflação de Variância (FIV) foi obtida para cada um dos coeficientes dos modelos de regressão múltipla por meio da expressão: $\operatorname{FIV}\left(\beta_{i}{ }_{i}\right)=1 /(1-$ $\mathrm{R}^{2}{ }_{i}$ ), em que: $\mathrm{R}^{2}{ }_{i}=$ coeficiente de determinação obtido pela regressão da variável explicativa $X_{i}(i=2, \ldots, \mathrm{p})$ com as demais variáveis independentes (SALMERÓN et al., 2013).

\section{Validação dos modelos selecionados}

Foi realizado o teste Qui-quadrado $\left(\chi^{2}\right)$ através da fórmula: $\chi^{2}=\Sigma\left(y_{i}-\hat{y}_{i}\right)^{2} / \hat{y}_{i}$, ao nível de $5 \%$ de probabilidade, utilizando-se do banco de dados independente constituído de 50 árvores. Em que: $y_{i}=$ altura real das árvores-amostras; $\hat{y}_{i}=$ altura estimada das árvoresamostras pelos modelos ajustados. Testou-se as seguintes hipóteses: Hipótese de nulidade: as alturas estimadas pelos modelos hipsométricos são estatisticamente iguais as alturas reais obtidas em campo; e Hipótese alternativa: as alturas estimadas pelos modelos hipsométricos são diferentes, estatisticamente, das alturas reais obtidas em campo.

\section{RESULTADOS E DISCUSSÃO}

A análise descritiva das variáveis dendrométricas utilizadas na modelagem da relação hipsométrica e na validação do modelo selecionado (Tabela 3), reporta elevada variabilidade nos valores de diâmetro das árvores-amostras de treino e validação. Contudo, a variável altura mostrou-se pouco heterogênea, com coeficiente de variação menor do que $10 \%$, tanto para os dados de treino quanto validação. De acordo com Rufino et al. (2010), uma maior heterogeneidade dos valores de altura e diâmetro certamente implicará em uma diminuição da correlação entre estas variáveis.

Azevedo et al. (2011) mencionam que povoamentos sem ocorrência de tratos silviculturais, por exemplo, desrama e desbaste, podem ser possíveis razões de um povoamento apresentar uma correlação muito baixa entre as variáveis diâmetro e altura, isto é, em povoamentos malconduzidos e formados ou em sítios não definidos, provavelmente haverá uma fraca correlação devido a maior heterogeneidade da população em estudo. $O$ povoamento em questão não foi devidamente conduzido, durante dois últimos anos, com falta de tratos silviculturais, como limpeza da área, poda e desrama, o que pode explicar a elevada variabilidade dos valores em torno da média, sobretudo, para a variável diâmetro.

Tabela 3. Estatística das variáveis dendrométricas para um povoamento híbrido (E. urophylla vs. $E$. grandis), no município de Pacajá, Pará

\begin{tabular}{|c|c|c|c|c|c|c|}
\hline & \multirow{2}{*}{ Variável } & \multicolumn{5}{|c|}{ Parâmetros } \\
\hline & & Média & Sd & CV (\%) & Mínimo & Máximo \\
\hline \multirow{2}{*}{$\stackrel{\stackrel{\oplus}{\frac{N}{2}}}{\frac{3}{4}}$} & Diâmetro & 14,25 & 3,70 & 25,97 & 4,46 & 23,87 \\
\hline & Altura & 19,29 & 1,63 & 8,47 & 12,00 & 22,80 \\
\hline 윰 & Diâmetro & 14,58 & 3,94 & 27,01 & 6,37 & 22,06 \\
\hline$\stackrel{.0}{\bar{D}}$ & Altura & 19,29 & 1,56 & 8,10 & 14,10 & 22,20 \\
\hline
\end{tabular}

As correlações das variáveis dependentes com as variáveis independentes e entre as variáveis independentes mostraram-se significativas ao nível de $1 \%$ de probabilidade $(\alpha=0,01)$, para todos os casos analisados (Tabela 4). A transformação da variável DAP para seu inverso e logaritmo neperiano ocasionou uma melhora na relação com a altura. No entanto, a variável altura transformada mostrou melhores correlações com às variáveis independentes, notadamente, na relação DAP e DAP/Vh, cujas variáveis estão constituídas no modelo Hiperbólico (modelo 9). Fato similar ocorreu na relação DAP e ${ }^{2}\left[D^{2} P^{2} /(h-1,3)\right]$, cujas variáveis estão constituídas no modelo Naslund (modelo 8 ) e também nas relações de DAP e DAP 2 com $\mathrm{DAP}^{2} / \mathrm{h}-1,3$ ou $\mathrm{DAP}^{2} / \mathrm{h}$, relativas aos modelos de Prodan (modelos 11 e 12, respectivamente).

Sanquetta et al. (2014) reportaram resultados semelhantes em seu estudo sobre relação hipsométrica em inventários pré-corte em povoamentos de Acacia mearnsii De Wild., ao observarem que a correlação da altura-diâmetro, em sua forma natural ou transformada, foi significativa ( $p \leq 0,05$ ), para todos os casos, afirmando que a transformação da variável diâmetro para seu inverso, inverso ao quadrado e logarítmica ocasionou uma melhora na relação com a altura.

Por outro lado, detectou-se alta correlação entre as variáveis independentes $D A P$ com $D A P^{2}$ e $L n D A P$ com $1 / D A P$ nas equações de Prodan (modelos 10 e 11) e Silva (1980) (modelo 17), respectivamente, fato não interessante em regressão, uma vez que, poderá ocasionar não significância nas estimativas dos coeficientes das equações devido à existência de multicolinearidade. Gonzáles e Formoso (2000) afirmam que na presença de correlação alta entre variáveis 
independentes, os coeficientes de regressão estimados tendem a ser imprecisos e as estimativas dos coeficientes podem oscilar consideravelmente.

Tabela 4. Correlação entre variáveis dependentes e independentes das equações hipsométricas ajustadas para um povoamento híbrido ( $E$. urophylla vs. E. grandis), no município de Pacajá, Pará

\begin{tabular}{|c|c|c|c|c|c|c|}
\hline & $D A P$ & $D A P^{2}$ & $\operatorname{Ln}(D A P)$ & $1 / D A P^{2}$ & $1 / D A P$ & $\log (D A P)$ \\
\hline$h$ & $0,74^{* *}$ & $0,69 * *$ & $0,78 * *$ & $-0,74 * *$ & $-0,79 * *$ & - \\
\hline $1 / \sqrt[3]{(h-1,3)}$ & - & - & - & - & $0,82^{* *}$ & - \\
\hline$D A P / V h$ & $0,99 * *$ & - & - & - & - & - \\
\hline $\operatorname{Ln}(h-1,3)$ & - & - & - & - & $-0,81 * *$ & - \\
\hline $\operatorname{Ln}(h)$ & - & - & $0,79 * *$ & - & $-0,81^{* *}$ & $0,79 * *$ \\
\hline$V(1 / h)$ & - & - & - & - & $0,82 * *$ & - \\
\hline$v\left[D A P^{2} /(h-1,3)\right]$ & $0,99 * *$ & - & - & - & - & - \\
\hline$D A P^{2} /(h-1,3)$ & $0,98 * *$ & $0,99 * *$ & - & - & - & - \\
\hline$D A P^{2} / h$ & $0,98 * *$ & $0,99 * *$ & - & - & - & - \\
\hline$\sqrt[3]{(1 / \operatorname{Ln} h)}$ & - & - & - & - & $0,82 * *$ & - \\
\hline$D A P$ & - & $0,99 * *$ & - & - & - & - \\
\hline 1/DAP & - & - & $-0,97 * *$ & - & - & - \\
\hline
\end{tabular}

** significativo ao nível de $1 \%$ de probabilidade $(\alpha=0,01)$.

$\mathrm{O}$ indício mais claro da existência da multicolinearidade é quando o coeficiente de determinação se mostra bastante alto, porém nenhum dos coeficientes da regressão é estatisticamente significativo, através do teste $t$. Maroco (2010) expõe que correlações bivariadas elevadas entre variáveis independentes $(r>0,75)$ conduzem geralmente a problemas de multicolinearidade.

Indícios da existência de multicolinearidade foram verificados para o modelo 9, 10, 11 e 17, tendo em vista que as variáveis independentes $D A P$ e $D A P^{2}, \operatorname{Ln}(D A P)$ e $1 / D A P$ mostraram-se altamente correlacionadas. Aliado a isso, os modelos de Prodan tiveram elevados valores de $\mathrm{R}^{2}$ aj. $\quad(0,98$ e 0,99, para os modelos 10 e 11 , respectivamente), porém, os coeficientes $\beta_{0}$ e $\beta_{1}$, foram não significativos através da estatística $t(\alpha=0,05)$. Silva (1980) teve o coeficiente $\beta_{1}$ não significante.

A estatística Fator de Inflação de Variância (FIV) ratifica a existência de efeitos graves da multicolinearidade sobre as estimativas dos coeficientes dos modelos de Trorey, Prodan (10 e 11) e Silva (1980), cujos valores do VIF foram superiores a 10 para os coeficientes da regressão. Portanto, estes quatro modelos tiveram sua adequabilidade para estimativa da altura rejeitada.

As estimativas dos coeficientes da regressão e suas significâncias através do teste $t$ e as estatísticas de ajuste e precisão dos modelos hipsométricos estão apresentadas nas Tabelas 5 e 6 , respectivamente. A estatística $F$ mostrou-se significativa ao nível de $1 \%$ de probabilidade para todos os modelos hipsométricos ajustados, rejeitando-se a hipótese de não haver regressão, isto é, conclui-se que pelo menos uma variável independente esteja relacionada com um valor da variável dependente. Os maiores valores do teste $\mathrm{F}$ foram observados para o modelo Hiperbólico (modelo 8), Prodan (modelo 10 e 11) e Naslund (modelo 7), respectivamente.

Os 17 modelos ajustados mostraram baixos valores de erro padrão de estimativa (Syx\%) variando entre 2,44 e $5,74 \%$. O modelo Hiperbólico teve menor valor de erro padrão (Syx\% = 2,44). Enquanto que, a maior parte dos modelos mostrou valores de erro-padrão superiores a $4 \%$. Os valores baixos de erro-padrão podem estar associados a baixa variabilidade da altura.

A maioria dos modelos ajustados apresentou valores de coeficiente de determinação ajustado ( $R^{2}$ aj.) inferiores a $70 \%$. Campos e Leite (2006) afirmaram que em se tratando de relações hipsométricas, é comum que o valor de $R^{2}$ aj. não ultrapasse $80 \%$, isto, porque, a correlação altura/diâmetro não é tão forte quanto à relação altura/volume.

Não obstante, para este estudo quatro modelos (Hiperbólico, Prodan [modelo 10 e 11] e Naslund) mostraram valores de $R^{2}$ aj. superiores a $90 \%$, indicando o elevado grau de explicação das variáveis dependentes pelas variáveis independentes dos modelos. Por outro lado, embora os modelos de Prodan (10 e 11), ainda que tenham mostrado boas estatísticas de precisão, sobretudo, quando comparado aos modelos de Assmann e Linear, tiveram os coeficientes da regressão $\beta_{0}$ e $\beta_{1}$ não significativos, através do teste $t \quad(\alpha=0,05)$, desconsiderando, assim, os modelos de Prodan para a estimativa da altura.

Rufino et al. (2010), estudando a relação hipsométrica em um povoamento de híbridos de $E$. urophylla conduzido sobre o sistema de rebrota após 7 anos no município de Niquelândia, no estado de Goiás, observaram valores baixos e semelhantes a este estudo para os modelos de Trorey (61\%), Linear (55\%) e Curtis (64\%). Batista et al. (2001) estudando a relação hipsométrica em um povoamento de E. grandis, aos seis anos de idade, no 
município de Anhembi, estado de São Paulo, observaram valores superiores de $\mathrm{R}^{2}$ aj. em relação a este estudo, para os modelos Linear $(73,4 \%)$, Trorey $(78,0 \%)$, Stoffels $(75,4 \%)$, Curtis $(78,8 \%)$ e Henriksen $(78,8)$, porém, para o modelo Hiperbólico o $\mathrm{R}^{2}$ aj. foi inferior $(78,7 \%)$.

Tabela 5. Coeficientes estimados dos modelos hipsométricos ajustados para um povoamento híbrido (E. urophylla vs. $E$. grandis), no município de Pacajá, Pará

\begin{tabular}{|c|c|c|c|c|c|}
\hline & Modelo & $\beta_{0}$ & $\beta_{1}$ & $\boldsymbol{\beta}_{2}$ & FIV \\
\hline 1 & Linear & $\begin{array}{c}14,7504 \\
(p=6,6 \mathrm{E}-155)\end{array}$ & $\begin{array}{c}0,3194 \\
(p=4,0 \mathrm{E}-49)\end{array}$ & - & - \\
\hline 2 & Petterson & $\begin{array}{c}0,2041 \\
(p=7,2 \mathrm{E}-296)\end{array}$ & $\begin{array}{c}0,3168 \\
(p=1,6 \mathrm{E}-69)\end{array}$ & - & - \\
\hline 3 & Petterson & $\begin{array}{c}0,3535 \\
(p=0)\end{array}$ & $\begin{array}{c}0,3775 \\
(p=3,5 \mathrm{E}-69)\end{array}$ & - & - \\
\hline 4 & Henriksen & $\begin{array}{c}8,1091 \\
(p=3,6 \mathrm{E}-37)\end{array}$ & $\begin{array}{c}4,2726 \\
(p=5,8 \mathrm{E}-58)\end{array}$ & - & - \\
\hline 5 & Assmann & $\begin{array}{c}20,5881 \\
(p=0)\end{array}$ & $\begin{array}{c}-198,1392 \\
(p=8,1 \mathrm{E}-50)\end{array}$ & - & - \\
\hline 6 & Curtis & $\begin{array}{c}0,1786 \\
(p=1,6 \mathrm{E}-41)\end{array}$ & $\begin{array}{c}2,2176 \\
(p=1,2 \mathrm{E}-262)\end{array}$ & - & - \\
\hline 7 & Naslund & $\begin{array}{c}0,3426 \\
(p=3,6 \mathrm{E}-197)\end{array}$ & $\begin{array}{c}0,2103 \\
(p=2,5 \mathrm{E}-198)\end{array}$ & - & - \\
\hline 8 & Hiperbólico & $\begin{array}{c}0,3071 \\
(p=3,8 \mathrm{E}-41)\end{array}$ & $\begin{array}{c}0,2048 \\
(p=1,1 \mathrm{E}-271)\end{array}$ & - & - \\
\hline 9 & Trorey & $\begin{array}{c}10,3461 \\
(p=9,0 \mathrm{E}-39)\end{array}$ & $\begin{array}{c}1,0004 \\
(p=1,2 \mathrm{E}-20)\end{array}$ & $\begin{array}{c}-0,0245 \\
(p=2,2 \mathrm{E}-11)\end{array}$ & 37,2213 \\
\hline 10 & Prodan & $\begin{array}{c}0,356 \\
(p=0,412)\end{array}$ & $\begin{array}{c}0,1051 \\
(p=0,103)\end{array}$ & $\begin{array}{c}0,0457 \\
(p=1,2 \mathrm{E}-55)\end{array}$ & 37,2213 \\
\hline 11 & Prodan & $\begin{array}{c}0,2755 \\
(p=0,473)\end{array}$ & $\begin{array}{c}0,0962 \\
(p=0,088)\end{array}$ & $\begin{array}{c}0,0431 \\
(p=4,4 \mathrm{E}-61)\end{array}$ & 37,2213 \\
\hline 12 & Pollanschutz & $\begin{array}{c}3,1049 \\
(p=0)\end{array}$ & $\begin{array}{c}-2,8676 \\
(p=4,8 \mathrm{E}-67)\end{array}$ & - & - \\
\hline 13 & Stoffels & $\begin{array}{c}2,3375 \\
(p=4,5 \mathrm{E}-193)\end{array}$ & $\begin{array}{c}0,2363 \\
(p=2,5 \mathrm{E}-60)\end{array}$ & - & - \\
\hline 14 & Curtis & $\begin{array}{c}3,1589 \\
(p=0)\end{array}$ & $\begin{array}{c}-2,6540 \\
(p=1,5 \mathrm{E}-66)\end{array}$ & - & - \\
\hline 15 & Petterson & $\begin{array}{c}0,6803 \\
(p=0)\end{array}$ & $\begin{array}{c}0,2177 \\
(p=2,5 \mathrm{E}-69)\end{array}$ & - & - \\
\hline 16 & Stoffels & $\begin{array}{c}2,3375 \\
(p=4,5 \mathrm{E}-193)\end{array}$ & $\begin{array}{c}0,5442 \\
(p=2,5 \mathrm{E}-60)\end{array}$ & - & - \\
\hline 17 & Silva (1980) & $\begin{array}{c}3,1411 \\
(p=3,4 \mathrm{E}-59)\end{array}$ & $\begin{array}{c}0,0052 \\
(p=0,905)\end{array}$ & $\begin{array}{c}-2,5991 \\
(p=9,8 \mathrm{E}-08)\end{array}$ & 17,0459 \\
\hline
\end{tabular}

$\beta_{0}, \beta_{1}, \beta_{2}$ e $\beta_{3}=$ coeficientes da regressão; $p$-value: indica o nível de significância do teste $t$ para cada coeficiente da regressão ( $\alpha$ $=0,05)$; e FIV = fator de inflação de variância.
Tabela 6. Medidas de precisão do ajustamento para um povoamento híbrido (E. urophylla vs. $E$. grandis), no município de Pacajá, Pará

\begin{tabular}{|c|c|c|c|c|c|c|c|}
\hline & Modelo & $\mathbf{F}$ & $\mathbf{R}_{\text {aj. }}$ & Syx (recalc.) & $\mathrm{S}_{\mathrm{yx}} \%$ & DMP\% & FM \\
\hline 1 & Linear & 330,75 & 0,54 & 1,06 & 5,48 & 8,92 & - \\
\hline 2 & Petterson & 576,16 & 0,67 & 0,93 & 4,81 & 0,06 & - \\
\hline 3 & Petterson & 571,34 & 0,67 & 0,93 & 4,80 & 0,07 & - \\
\hline 4 & Henriksen & 426,85 & 0,61 & 0,97 & 5,01 & 0,27 & - \\
\hline 5 & Assmann & 337,82 & 0,55 & 1,11 & 5,74 & 0,28 & - \\
\hline 6 & Curtis & 451,42 & 0,62 & 0,93 & 4,84 & 0,25 & - \\
\hline 7 & Naslund & 21073,18 & 0,99 & 0,93 & 4,81 & 0,07 & - \\
\hline 8 & Hiperbólico & 24549,27 & 0,99 & 0,08 & 2,44 & 0,08 & - \\
\hline 9 & Trorey & 218,29 & 0,61 & 0,98 & 5,06 & 0,27 & - \\
\hline 10 & Prodan & 8719,87 & 0,98 & 0,93 & 4,83 & $-0,03$ & - \\
\hline 11 & Prodan & 10069,86 & 0,99 & 0,93 & 4,83 & $-0,01$ & - \\
\hline 12 & Pollanschutz & 541,62 & 0,66 & 0,99 & 5,13 & 1,57 & 1,0145 \\
\hline 13 & Stoffels & 455,21 & 0,62 & 1,06 & 5,50 & 1,54 & 1,0140 \\
\hline 14 & Curtis & 534,9 & 0,66 & 0,97 & 5,05 & 1,38 & 1,0126 \\
\hline 15 & Petterson & 573,33 & 0,67 & 0,93 & 4,80 & 0,08 & 1,0001 \\
\hline 16 & Stoffels & 455,21 & 0,62 & 1,06 & 5,50 & 1,54 & 1,0140 \\
\hline 17 & Silva (1980) & 266,5 & 0,66 & 0,98 & 5,06 & 1,39 & 1,0126 \\
\hline \multicolumn{8}{|c|}{$\begin{array}{l}\mathrm{F}=\text { estatística F-Snedecor da ANOVA de regressão; } \mathrm{R}^{2} \text { aj. } \\
\text { coeficiente de determinação ajustado; Syx(recalc.) = erro padrão da } \\
\text { estimativa recalculado, em metros; Syx\% = erro padrão da } \\
\text { estimativa recalculado, em porcentagem; DMP\% = desvio médio } \\
\text { percentual; e FM = fator de correção de Meyer. }\end{array}$} \\
\hline
\end{tabular}

Moraes Neto et al. (2010) observaram valores superiores de $\mathrm{R}^{2} \mathrm{aj}$. aos deste estudo, para o modelo Linear $(80,0 \%$ e $87,0 \%)$, Trorey $(81,0 \%$ e $88,0 \%)$, Stoffels $(84,0 \%$ e $90,0 \%)$ e Henriksen $(77,0 \%$ e $84,0 \%)$, para os povoamentos de Eucalyptus cloeziana e de híbrido de E. urophylla e grandis, respectivamente, aos 18 meses de idade, em Planaltina, Distrito Federal, e o $\mathrm{R}^{2} \mathrm{aj}$. foi inferior para o modelo Assmann $(32,0 \%$ e $25,0 \%)$ e semelhante para o modelo Hiperbólico ( $R^{2}$ aj. $=93,0 \%$ e $95,0 \%$ ), respectivamente.

Sousa et al. (2013) estudando a relação hipsométrica para povoamentos de E. urophylla sob alto fuste $\mathrm{e}$ talhadia aos 5 anos de idade numa fazenda do distrito de Pradoso pertencente ao município de Vitória da Conquista, Bahia, observaram nos plantios sob alto fuste valores inferiores de $\mathrm{R}^{2} \mathrm{aj}$. para os modelos Linear $(40,9 \%)$, Henriksen (43,3\%), Assmann (40,2\%), Trorey $(40,2 \%)$, Prodan $(81,9 \%)$, Curtis $(43,1 \%)$, Stoffels $(43,1 \%)$ e Silva (1980) $(41,4 \%)$, em relação a este estudo. 
Nos Plantios sob talhadia, observaram valores inferiores para os modelos Henriksen (17,9\%), Curtis $(55,2 \%)$ e Silva (1980) $(54,7 \%)$, enquanto que houve semelhanças para o modelo Linear $(55,6 \%)$, Assmann $(50,8 \%)$, Trorey $(54,4 \%)$, Prodan $(93,8 \%)$ e Stoffels $(56,1 \%)$. Curto et al. (2014) observaram valor inferior para o modelo Naslund (74,8\%), para uma floresta estacional semidecidual no município de Guaçuí, estado de Espírito Santo. De maneira geral, foi possível detectar nos estudos citados a superioridade dos modelos de Prodan e Hiperbólico e a inferioridade dos modelos de Linear, Assmann e Trorey, em termos de coeficiente de determinação ajustado, resultados que corroboram com este estudo.

Santos et al. (2014) estudando a relação hipsométrica de um povoamento do mesmo híbrido ( $E$. urophylla e $E$. grandis) aos seis anos de idade, no município de Mojú no Estado do Pará, observaram valores inferiores de $\mathrm{R}^{2}$ aj. em relação a este estudo para o modelo Linear (28,68\%), Assmann (29,35\%), Trorey (28,88\%), Henriksen (29,52\%) Curtis (31,57\%), Stofells (30,91\%), Prodan (79\%) e Hiperbólico (80,60\%). Porém, similarmente ao observado para hibrido de oito anos deste estudo, o modelo Hiperbólico mostrou-se superior.

Resultados semelhantes a este estudo foram identificados para outras espécies florestais, não pertencentes ao gênero Eucalyptus. Sanquetta et al. (2014) estudando a relação hipsométrica em um povoamento de híbridos de Acacia mearnsii De Wild, aos sete anos de idade conduzido sobre o sistema de rebrota nos municípios de Cristal e Piratini no Rio Grande do Sul observaram valores baixos e semelhantes de $R^{2} a j$. a este estudo para os modelos de Curtis (61\%) e Stofells (56\%). Rossi et al. (2011), para povoamentos de Tectona grandis L. F. entre 1 e 5 anos e 26 anos de idade, no município de Monte Dourado, Pará, constataram valores de $\mathrm{R}^{2} a j$. análogos para o modelo de Curtis (66\%).

Barros et al. (2002) estudando Plantações de Pinus oocarpa de idades entre 5 e 25 anos no município de Agudos do Estado de São Paulo, encontraram valores similares para os modelos Henriksen (63,80\%), Trorey $(63,66 \%)$, Curtis [modelos $6(62,80 \%)$ e 15(64,95\%)], Stofells $(63,9 \%)$ e Linear (59,84\%). Araújo et al. (2012b) ajustando modelos hipsométricos para plantios desde a década de 1970 de Pinus sp. nos municípios de Urucaia e Chapada Gaúcha no estado de Minas Gerais, também constataram valores de $\mathrm{R}^{2} \mathrm{aj}$. similares a este estudo para os modelos Petterson (71,6\%) e Naslund $(92,5 \%)$.

Os estudos de relação hipsométrica para o gênero Eucalyptus mencionados anteriormente (MORAES NETO et al., 2010; RUFINO et al., 2010; SOUSA et al., 2013) reportaram valores superiores de Syx\% para todos os modelos comuns a este estudo. Moraes Neto et al. (2010) revelaram dados de Syx\% que variaram entre 10,0\% e $30,0 \%$. Sousa et al. (2013) observaram variação entre $5,0 \%$ e $10,0 \%$ para Syx\%. Rufino et al. (2010) notaram erro padrão de estimativa entorno de $10,0 \%$.

Os valores de desvio médio percentual (DMP\%) mostraram-se altamente dispersos entre os modelos ajustados, variando entre $-0,03 \%$ a $8,92 \%$. Os modelos de Petterson (2, 3 e 15), Prodan (10 e 11), Naslund e Hiperbólico mostraram melhores resultados de DMP, cujos valores foram abaixo de $\pm 1 \%$. Além disso, a distribuição dos resíduos para estes modelos mostrou-se mais homogênea e próxima da média zero.

Ademais, as análises da distribuição dos resíduos da maioria dos modelos ajustados mostraram uma leve heterogeneidade, superestimando mais nas primeiras classes de diâmetros, sendo mais acurado nas ultimas classes. O modelo Linear apresentou uma tendência sistemática negativa muito expressiva na distribuição de seus resíduos, superestimando o volume nas primeiras classes de diâmetro (Figura 1).

No que diz respeito à distribuição dos resíduos, os estudos reportados anteriormente para o gênero Eucalyptus mostraram resultados semelhantes a este trabalho. Moraes Neto et al. (2010) constataram que dentre outros o hiperbólico foi um dos modelos que melhor aderiu ao eixo horizontal no gráfico de distribuição dos resíduos para o híbrido ( $E$. urophylla e $E$. grandis) e para Eucalyptus cloeziana, enquanto que os modelos polinomiais a exemplo do Linear e Trorey estão entre os modelos que apresentaram maiores dispersões dos resíduos.

De acordo com Batista et al. (2001), a representação da curvatura da relação altura-diâmetro por estes modelos é bastante artificial, com extrapolação ligeiramente fora da amplitude da amostra, resultando em grandes erros nas estimativas. Rufino et al. (2010) também identificaram uma leve tendenciosidade em superestimar os valores observados de DAP e altura, sendo esta tendenciosidade mais acentuada nos modelos de Stoffels e Linear. Sousa et al. (2013) reportaram para os modelos selecionados (Henriksen, Trorey, Prodan, Stoffels) boa distribuição gráfica dos resíduos em torno da média, porém, diferentemente ao observado neste estudo, não verificaram a ocorrência de tendências em superestimar ou subestimar a variável altura total em função da variável DAP. 

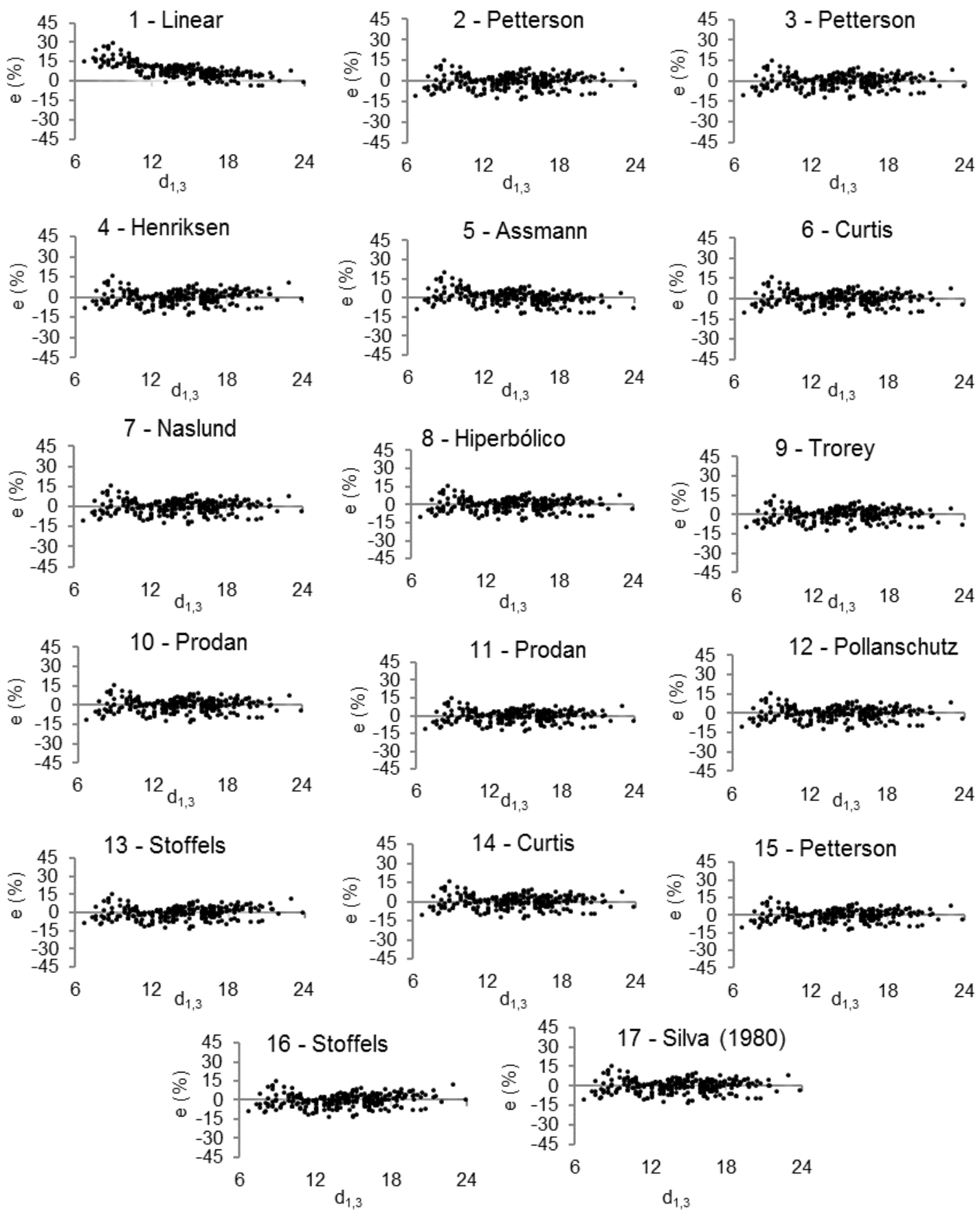

Figura 1. Distribuição de resíduos em função do diâmetro a $1,3 \mathrm{~m}$ do solo, em centímetros, um povoamento híbrido ( $E$. urophylla vs. E. grandis), no município de Pacajá, Pará.

Assim, considerando-se a significância dos coeficientes da regressão e as melhores medidas de qualidade de ajuste e uma distribuição de resíduos aleatória e homogênea em torno da média zero, o modelo Hiperbólico e de Naslund foram considerados mais satisfatórios para as estimativas de altura total do povoamento híbrido (E. urophylla e E. grandis).

Adicionalmente, o teste Qui-quadrado $\left(\chi^{2}\right)$ foi aplicado ao conjunto de árvores-amostras independentes (50), com fins de comparar os valores estimados de altura por ambos os modelos (Hiperbólico e Naslund) em relação aos valores reais de campo. Assim, a estatística $\chi^{2}$ revelou que as alturas reais não diferem estatisticamente dos respectivos valores das alturas estimadas pelas modelos Hiperbólico e Naslund.

Donadoni et al. (2010) reportaram resultados semelhantes a este estudo ao selecionarem o modelo hiperbólico, como melhor modelo para estimar a altura nos povoamentos de Pinus caribaea var. hondurensis ( $R^{2}$ aj. $=89,60 \%$ e Syx\% $\left.= \pm 3,94 \%\right)$, e Pinus tecunumanii 
( $R^{2}$ aj. $=97,3 \%$ e Syx\% = \pm 6,32\%), no município de Vilhena, estado de Rondônia com ambos possuindo 12 anos de idade.

Moraes Neto et al. (2010) indicaram o modelo hiperbólico como um dos melhores para estimar a altura do híbrido E. urophylla com E. grandis e E. cloeziana, aos 18 meses na Embrapa Cerrado em Planaltina, Distrito Federal. Santos et al. (2014) estudando a relação hipsométrica em um plantio do híbrido (E. urophylla e $E$. grandis) aos seis anos de idade no município de Mojú no Estado do Pará escolheram o modelo Hiperbólico como um dos melhores modelos. Araújo et al. (2012b) optaram pelo modelo Naslund como melhor equação para estimar a altura de Pinus sp. em plantios estabelecidos desde a década de 1970 nos municípios de Urucaia e Chapada Gaúcha no estado de Minas Gerais.

De modo divergente, Soares et al. (2004) ao realizarem ajustes para um povoamento de $E$. grandis W. Hill ex Maiden com idades variando entre 4 e 7,1 anos de idade, em Guatapará, São Paulo, selecionaram o modelo de Stoffels $[\ln (h)=1,56207+0,56904 \operatorname{Ln}(D A P)]$, tendo em vista as melhores medidas de qualidade de ajuste $\left(\mathrm{R}^{2} \mathrm{aj} .=\right.$ $72,98 \%$, Syx $= \pm 2,0 \mathrm{~m} ; \pm 10,1 \%$ ). Rocha et al. (2010) reportaram em seu estudo com $E$. urophylla com 5 anos de idade, no Planalto da Conquista, Bahia, que os modelos Trorey e Prodan (modelo 9 e 11) apresentaram boas estatísticas de ajuste para estimativa da altura.

Rufino et al. (2010) citaram o modelo de Trorey ( $\mathrm{h}=$ $5,92481+3,38051 \mathrm{DAP}-0,09787 \mathrm{DAP}^{2}$ ) como sendo aquele com melhores ajustes em povoamento de $E$. urophylla conduzido sob regime de rebrota após 7 anos ( $R^{2}$ aj. $=60,56 \%$, Syx $= \pm 2,14 \mathrm{~m} ; \pm 10,16 \%$ ). Sousa et al. (2013) indicaram os modelos de Henricksen e Trorey para a estimativa da variável altura total em povoamento de $E$. urophylla conduzido sob regime de alto fuste e Prodan (modelo 11) em povoamento conduzido sob regime de talhadia em Vitória da Conquista, Bahia, aos 5 anos de idade.

De modo geral, constatou-se superioridade dos modelos deste estudo, em termos de medidas de qualidade de ajuste, em comparação com as equações hipsométricas selecionadas por outros estudos. Por exemplo, os modelos de Trorey, Stoffels, Henriksen e Trorey selecionados nos estudos de Rufino et al. (2010), Soares et al. (2004) e Sousa et al. (2013), respectivamente, mostraram valores de $\mathrm{R}^{2}$ aj. e Syx (\%) inferiores aos mesmos modelos ajustados neste estudo. Por outro lado, ressalta-se que os estudos supracitados não ajustaram os modelos Hiperbólico e Naslund.

\section{CONCLUSÕES}

Os modelos Hiperbólico e Naslund, respectivamente $V D A P^{2} /(h-1,3)=0,3071+0,2048 D A P$ e $D A P / v h=0,3426+0,2103 D A P$, mostraram as melhores medidas de qualidade de ajuste para a relação altura-diâmetro, com pequena vantagem para o Hiperbólico devido ao seu menor valor de erro padrão de estimativa. Portanto, ambos os modelos se mostraram apropriados para a estimativa da altura do híbrido (E. urophylla e E. grandis).

\section{REFERÊNCIAS}

ABRAF. Anuário estatístico da ABRAF 2013, ano base 2012. Brasília: ABRAF, 2013. 142 p.

ARAÚJO, E. J. G.; PELISSARI, A. L.; DAVID, H. C.; SCOLFORO, J. R. S.; NETTO, S. P.; MORAIS, V. A. Relação hipsométrica para candeia (Eremanthus erythropappus) com diferentes espaçamentos de plantio em Minas Gerais, Brasil. Pesquisa florestal brasileira, v. 32, n. 71, p. 257-268, 2012a.

ARAÚJO, E. J. G.; PELISSARI, A. L.; DAVID, H. C.; MIRANDA, R. O. V.; NETTO, S. P.; MORAIS, V. A.; SCOLFORO, J. R. S. Relações dendrométricas em fragmentos de povoamentos de pinus em Minas Gerais. Pesquisa florestal brasileira, v. 32 , n. 72 , p. 355366, 2012b.

AZEVEDO, T. L.; MELLO, A. A.; FERREIRA, R. A.; SANQUETTA, C. R.; NAKAJIMA, N. Y. Equações hipsométricas e volumétricas para um povoamento de Eucalyptus sp. localizado na FLONA do Ibura, Sergipe. Revista Brasileira de Ciências Agrárias, v. 6, n. 1, p. 105112, 2011.

BARROS, D. A.; MACHADO, S. A.; ACERBI JUNIOR, F. W.; SCOLFORO, J. R. S. Comportamento de modelos hipsométricos tradicionais e genéricos para plantações de Pinus oocarpa em diferentes tratamentos. Boletim de Pesquisa Florestal, n. 45. p. 3-28, 2002.

BATISTA, J. L. F.; COUTO, H. T. Z.; FILHO, D. F. S. Quantificação de recursos florestais: árvores, arvoredos e florestas. 1 ed. São Paulo: Oficina de Textos, 2014.

BATISTA, J. L.; COUTO, H. T. Z.; MARQUESINI, M. Desempenho de modelos de relações hipsométricas: estudo em três tipos de floresta. Scientia Forestalis, n. 60, p. 149-163, 2001.

CAMPOS, J. C. C.; LEITE, H.; G. Mensuração Florestal: Perguntas e Respostas. 2 ed. Viçosa: UFV, 2006.

CURTO, R. A.; LOUREIRO, G. H.; MÔRA, R.; MIRANDA, R. O. V.; NETTO, S. P.; SILVA, G. F. Relações hipsométricas em floresta estacional semidecidual. Revista de Ciências Agrárias, v. 57, n. 1, p. 57-66, 2014.

DONAdONI, A. X.; PELISSARI, A. L.; DRESCHER, R.; ROSA, G. D. Relação hipsométrica para Pinus caribaea var. hondurensis e Pinus tecunumanii em povoamento homogêneo no Estado de Rondônia. Ciência Rural, v. 40, n. 12, p. 2499-2504, 2010. 
INSTITUTO BRASILEIRO DE GEOGRAFIA E ESTATÍSTICA (IBGE). Arquivo Shapefile de dados de pedologia da Amazônia Brasileira. 2012. Disponível em: <ftp://geoftp.ibge.gov.br/ mapeamento sistematico/banco dados georeferenciado recur sos naturais/amazonia legal/pedologia/pedologia.zip>. Acesso em: 06/11/2014.

INSTITUTO DE DESENVOLVIMENTO ECONOMICO, SOCIAL E AMBIENTAL DO PARÁ (IDESP). Cadeias de comercialização de produtos Florestais Não Madeireiros na Região de Integração Xingu, Estado do Pará: Relatório Técnico. Belém, 2011.

GONZÁLES, M. A. S.; FORMOSO, C. T. Análise conceitual das dificuldades na determinação de modelos de formação de preços através de análise de regressão. Revista Engenharia Civil, n. 8, p. 65-75, 2000.

MAROCO, J. Análise Estatística - Com utilização do SPSS. 3 ed. Lisboa, 2010. 824 p.

MORAES NETO, S. P.; PULROLNIK, K.; VILELA, L.; MUNHOZ, D. J. M.; GUMARÃES JÚNIOR, R.; MARCHÃO, R. L. Modelos hipsométricos para Eucalyptus cloeziana e Eucalyptus grandis em Sistemas Agrossilvipastoril. Planaltina, DF: Embrapa Cerrados, 2010.

OLIVEIRA, F. G. R. B.; SOUSA, G. T. O.; AZEVEDO, G. B.; BARRETO, P. A. B. Desempenho de modelos hipsométricos para um povoamento de Eucalyptus urophylla no município de Jaguaquara, Bahia. Enciclopédia Biosfera, v. 7, n. 13. p. 331-338, 2011.

RIBEIRO, A.; FILHO, A. C. F.; MELLO, J. M.; FERREIRA, M. Z. Estratégias e metodologias de ajuste de modelos hipsométricos em plantios de Eucalyptus sp. Cerne, v. 16, n. 1, p. 22-31, 2010.

ROCHA, T. B.; CABACINHA, C. D.; ALMEIDA, R. C.; PAULA, A.; SANTOS, R. C. Avaliação de métodos de estimativa de volume para um povoamento de Eucalyptus urophylla S. T. Blake no Planalto da Conquista- BA. Enciclopédia Biosfera, v. 6, n. 10. p. 1- 13, 2010.

ROSSI, A. S.; DRESCHER, R.; PELISSARI, A. L.; LANSSANOVA, L. R. Relação hipsométrica e crescimento de Tectona grandis L. f. no município de Monte Dourado, Pará. Scientia Forestalis, v. 39, n. 91, p. 301-307, 2011.

RUFINO, R. F.; MIGUEL, E. P.; SANTOS, G. A.; SANTOS, T. E. B.; SOUZA, F. Ajuste de modelos hipsométricos para um povoamento de eucaliptos conduzido sobre o sistema de rebrota. Enciclopédia Biosfera, v. 6, n. 10, p. 1-10, 2010.

SALMERÓN, R.; GARCÍA, C.; LÓPEZ, M. M. A note about the Variance Inflaction Factor and the Ridge Regression. Conference of Informatics and Management Sciences, p. 197- 199. 2013.

SANQUETTA, C. R.; BEHLING, A.; CORTE, A. P. D.; RUZA, M. S.; SIMON, A.; De SÃO JOSÉ, J. F. B. Relação hipsométrica em inventários pré-corte em povoamentos de Acacia mearnsii De Wild. Científica, v. 42, n. 1, p. 80-90, 2014.

SANQUETTA, C. R.; WATZALAWICK, L. F.; CORTE, A. P. D.; FERNANDES, L. A. V.; SIQUEIRA, J. D. P. Inventários Florestais:
Planejamento e Execução. 2 ed. Curitiba: Multi-Graphic Gráfica e Editora, 2009. 316 p.

SANTOS, M. L.; BRASIL NETO, A. B.; PAUMGARTTEN, A. É. A.; RODRIGUES, R. P.; SANTOS, C. R. Estimativa da relação hipsométrica para um povoamento de Eucalyptus "urograndis" no município de Mojú, nordeste paraense. Enciclopédia Biosfera, v. 10, n. 19. p. 1039-1048, 2014.

SOARES, T. S.; SCOLFORO, J. R. S.; FERREIRA S. O.; MELLO, J. M. Uso de diferentes alternativas para viabilizar a relação hipsométrica no povoamento florestal. Revista Árvore, v. 28, n. 6. p. 845-854, 2004.

SOUSA, G. T. O.; AZEVEDO, G. B.; BARRETO, P. A. B.; JÚNIOR, V. C. Relações hipsométricas para Eucalyptus urophylla conduzidos sob regime de alto fuste e talhadia no Sudoeste da Bahia. Scientia Plena, v. 9, n. 4., p 1-7, 2013.

VALENTE, M. D. R.; QUEIROZ, W. T.; PINHEIRO, J. G.; MONTEIRO, L. A. S. Modelo de predição para o volume total de quaruba (Vochysia inundata Ducke) via análise de fatores e regressão. Revista Árvore, v. 35, n. 2, p. 307-317, 2011. 